\title{
A PROSPECTIVE STUDY ON PIOGLITAZONE EFFECT IN REDUCING TRIGLYCERIDE LEVEL IN TYPE-2 DIABETIC PATIENTS
}

\section{Pharmacology}

\author{
Dr. R. Vijayarani*
}

M.D DLO, Assistant Professor, Department of Pharmacology, Government Sivagangai Medical college, Sivagangai. *Corresponding Author

\section{Tarunika. K. M II MBBS Student, Government Sivagangai Medical college, Sivagangai}

\section{ABSTRACT}

Type 2 diabetes mellitus is the most common form of diabetes mellitus characterized by hyperglycemia, insulin resistance, and relative insulin deficiency Dyslipidemia being common with diabetes, if we have a drug which can take care of both diabetes and Dyslipidemia, it will be more economical as well as increases the patient compliance. There are many studies on the effect of pioglitazone on triglyceride level. So this study was an attempt to find the effect of pioglitazone in diabetic patients with dyslipidemia in rural population. History taking and investigations were taken in 30 out patients visiting government sivagangai medical hospital between May 2019 and June 2019 The pre-treatment FBS mean was found to be $149.4+41.5$ and the post treatment mean was found to be $98.2+27.3$. Thus there is a significant reduction in fasting blood sugar level with $\mathrm{p}$ value less than 0.05 . Serum triglycerides investigation pre treatment mean was found to be $181.5+18.2$ and the post treatment mean is $160.07+21.03$ .Thus there is a significant decrease of $\mathrm{p}<0.05$ when pre and post treatment mean values were compared. The effect of pioglitazone on triglyceride level in type 2 diabetic patients was studied, it is evident that the Pioglitazone is both antidiabetic and antiatherogenic drug; we can conclude that the pioglitazone administration is effective to bring about significant change in the blood sugar, triglycerides. pioglitazone is a safe drug with less of side effects. , it will be more economical as well as increase patient compliance.

\section{KEYWORDS}

\section{Diabetes, dyslipidemia, Pioglitazone, Triglycerides}

\section{INTRODUCTION}

Diabetes mellitus is one of the oldest diseases known to man. It was first reported in Egyptian manuscript about 3000 years ago [1]. In 1936, the distinction between type 1 and type 2 diabetes mellitus was clearly made [2] Type 2 diabetes mellitus was first described as a component of metabolic syndrome in 1988 [3].Type 2 diabetes mellitus is the most common form of diabetes mellitus characterized by hyperglycemia, insulin resistance, and relative insulin deficiency[4] Type 2 diabetes mellitus results from interaction between genetic, environmental and behavioral risk factors People living with type 2 diabetes mellitus are more vulnerable to various forms of both short- and long-term complications, which often lead to their premature death. Diabetes Mellitus is common in Indian population?. Diabetes has become potential epidemic in India with more than 62 million diabetic individuals currently diagnosed with the disease. In 2000, India (31.7 million) topped the world with the highest number of people with diabetes mellitus followed by China (20.8 million) with the United States (17.7 million) in second and third place respectively. The incidence of type 2 diabetes is at epidemic proportions throughout the world. Patients with diabetes have a 2-4 fold increased risk of cardiovascular disease when compared to the general population. They also have a greatly increased risk for micro vascular disease. Type 2 diabetes is associated with an increased risk of cardiovascular disease (CVD) A major reason for this increased risk is Dyslipidemia, which is characterized by low HDL cholesterol (HDL-C), raised triglycerides (TGs) and a predominance of small dense LDL cholesterol (LDL-C) particles. In patients with Type 2 diabetes, even when in good glycemic control, there are abnormalities in lipid levels.

It is estimated that $30-60 \%$ of patients with Type 2 diabetes have dyslipidemia Specifically, patients with Type 2 diabetes often have an increase in serum triglyceride level, increased VLDL and LDL, and decreased HDL cholesterol levels. Non-HDL cholesterol levels are increased due to the increase in VLDL and LDL. LDL cholesterol levels are typically not different than in normal subjects but there is an increase in small dense LDL, a lipoprotein particle that may be particularly pro-atherogenic. As a consequence there are more LDL particles, which coupled with the increase in VLDL and LDL, leads to an increase in Apo B Studies have shown that the anti-oxidant and anti-inflammatory functions of HDL isolated from patients with diabetes are reduced; indicating that HDL levels per se may not fully reflect risk Oral hypoglycemic agents are used to reduce the blood sugar level in Type II diabetic patients. They are indicated if lifestyle modifications such as weight loss, dietary modifications, and exercise, do not control diabetes sufficiently. One of the oral anti diabetic drug is Pioglitazone which belongs to Thiazolidinediones group. Thiazolidinediones (TZD) are one such class of drugs that work through PPAR gamma activation. Pioglitazone is a TZD which is widely used for treating patients with type 2 diabetes. Pioglitazones also lower triglycerides and increase HDL cholesterol without affecting total cholesterol and LDL cholesterol Pioglitazone is an antidiabetic drug that, in the presence of insulin resistance, it increases hepatic and peripheral insulin sensitivity, thus inhibiting hepatic gluconeogenesis and increasing peripheral and splanchnic glucose uptake. Dyslipidemia being common with diabetes, if we have a drug which can take care of both diabetes and Dyslipidemia, it will be more economical as well as increases the patient compliance. There are many studies on the effect of pioglitazone on triglyceride level. So this study was an attempt to find the effect of pioglitazone in diabetic patients with dyslipidemia in rural population.

\section{AIM}

To study the effect of pioglitazone on triglyceride level in type 2 diabetic patients with Dyslipidemia.

\section{MATERIALAND METHODS}

Study Center: Government Medical College, Sivagangai, Tamilnadu Study Department: Department of Pharmacology

Supporting Department - Department of Medicine and department of Biochemistry

Study Duration: 2 months

Study Design: Prospective open labeled single center observational study

Sample size: 30 patients

Ethical Clearance: Institutional ethical clearance was obtained

Informed Written Consent: obtained from all the patients

Selection Of Participants:

\section{Inclusion Criteria:}

Sex: both male and female patients

Age group : 45-70

- Patient : Newly diagnosed type II Diabetic mellitus patients with associated hyperlipidemia

\author{
Exclusion Criteria: \\ Type IDM patients \\ Type II DM patients on other anti diabetic agents \\ - Patients with anemia \\ Patients with cardiac disorders, liver dysfunction and renal failure \\ Pregnant and lactating women \\ Hypertensive patients \\ Patient not willing to participate
}

Withdrawal Criteria: if any patients developing adverse drug effect during the study will be removed from the study.

Study Drug: tablet pioglitazone $15 \mathrm{mg}$ once a day after food 
Manufacturing date-04/2019

Expiry date- 03/2021

Batch number- ESU0701

Company-Sun Pharma Laboratories

\section{METHODOLOGY:}

Participants satisfying the inclusion and exclusion criteria were selected from the outpatient department of medicine and informed written consent was obtained from them. Participation was purely voluntary in nature and they were allowed to quit the study whenever they want or if they develop any serious adverse reaction. Selected participants were subjected to history taking; general examination systemic examination and base line investigation were done. The base line investigations were hemoglobin, complete blood count, blood urea, serum creatinine, liver function test, fasting blood sugar, post prandial blood sugar, lipid profile. Blood of diabetic patients were sampled from the antecubital vein after an overnight fast and the data were entered into the proforma designed for the study. Then the patients were given drug pioglitazone $15 \mathrm{mg}$ od for a period of two weeks and they were asked to come for review once in 15 days .In the review the patients clinical condition and any adverse effects if patients developed were enquired and the drug was given for next 15 days. After completion of the study the base line investigations were repeated for all the patients.

\section{Data Collection:}

Data collected were entered in the excel master sheets and that was subjected to statistical analysis

\section{Statistical Analysis}

Data were analyzed using spss version 16 software. Data were expressed as mean \pm SD using descriptive statistics. Parametric test paired student $t$ test was used to compare the pre test and post test and $p$ value $<0.05$ was considered statistically.

\section{OBSERVATIONS AND RESULTS}

History taking and investigations were taken in 30 out patients visiting government sivagangai medical hospital between May 2019 and June 2019. The data was collected and analyzed. Among the 30 patients, 12 were males and 18 were females. They were given tab. Pioglitazone once a day for a period of 2 months.

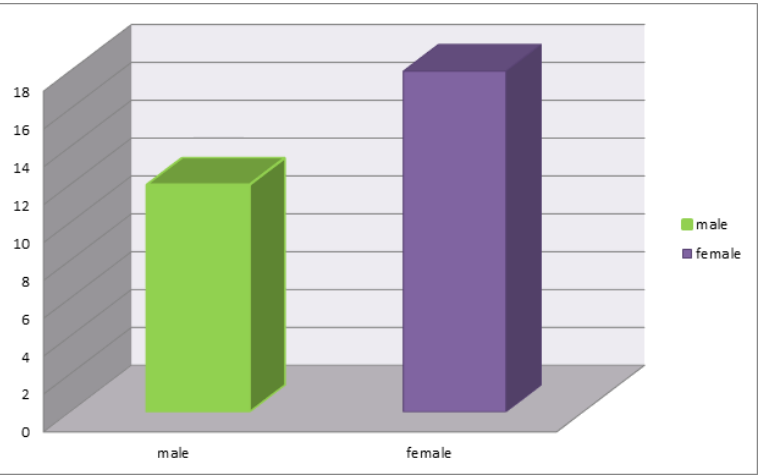

Figure1: Sex Distribution

\section{Age Distribution}

Out patients with diabetic dyslipidemia of age group 40 to 70 were included in the study after getting informed consent. This age group is mostly diagnosed with diabetes and dyslipidemia.

\section{Blood Sugar Level}

Blood sugar investigations were done before and after treatment which included fasting and post prandial blood sugar.

The pre-treatment FBS mean was found to be $149.4 \pm 41.5$ and the post treatment mean was found to be $98.2 \pm 27.3$. Thus there is a significant reduction in fasting blood sugar level with $\mathrm{p}$ value less than 0.05 .

The pre-treatment PPBS mean value is $232.8+47.8$ and the post treatment mean was found to be $163.9 \pm 38.7$. The pre and post treatment value were compared and was found to have a significant decrease of $\mathrm{p}<0.05$.

Thus there is an overall significant decrease in the blood sugar of $\mathrm{p}<0.05$.

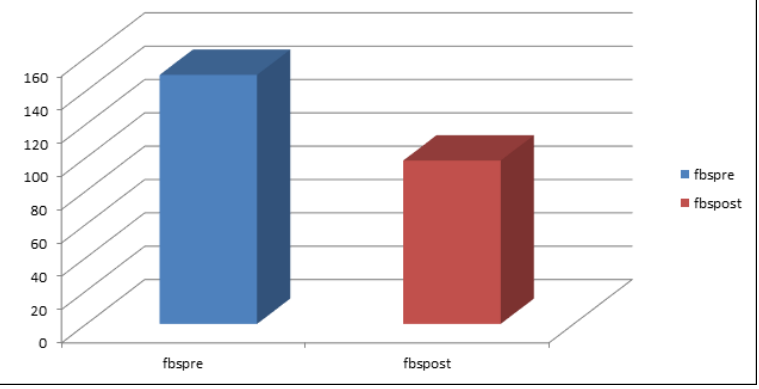

Figure2: Comparision Of Fasting Blood Sugar Level Pre And Post Treatment

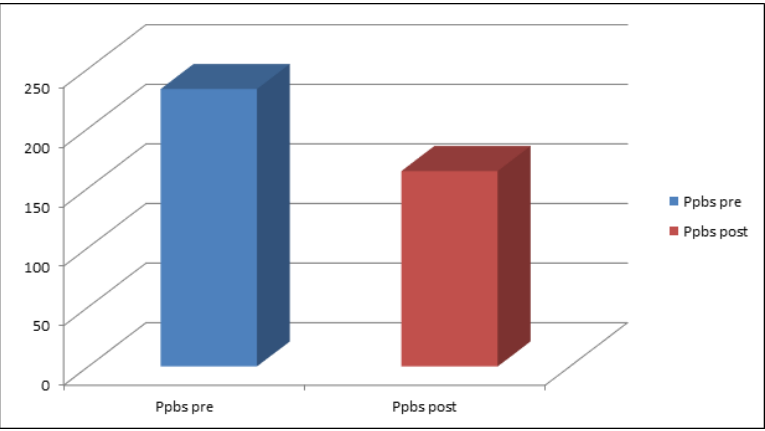

Figure 3: Comparision Of Post Prandial Blood Sugar Level Pre And Post Treatment

Serum triglycerides investigation pre treatment mean was found to be $181.5 \pm 18.2$ and the post treatment mean is $160.07 \pm 21.03$. Thus there is a significant decrease of $p<0.05$ when pre and post treatment mean values were compared.

Table 5: Comparision Of Triglyceride Level Pre And Post Treatment

\begin{tabular}{|l|l|l|l|}
\hline TRIGLYCERIDES & MEAN & STD. DEVIATION & P VALUE \\
\hline Pre treatment & 181.5 & 18.2 & P $<0.05$ \\
significant
\end{tabular}

History taking ( general \& family ), examination ( general \& systemic examination ) and investigations ( blood sugar, lipid profile, serum creatinine and serum urea ) were taken for the out patients. Among the out patients, 30 diabetic patients with dyslipidemia were enrolled in the study according to the inclusion and exclusion criteria, after getting their informed consent.Among the 30 patients, 12 were males and 18 were females. It is noted that the diabetic females with dyslipidemia are 1.5 folds more than the males. The difference between male and female also depends upon the life style and hormonal pattern In our study we found diabetic patients with dyslipidemia were in the age group 40-70.Diabetic patients mostly fall between the age group 40 to 70. These patients were administrated with pioglitazone $15 \mathrm{mg}$ OD for a period of two months. After a period of two months, blood investigations were taken and the results were obtained. The pre treatment results were compared with the post treatment result using paired student $t$ test. There are many antidiabetic drugs available for the treatment. They were Sulfonylureas (glipizide, glyburide, gliclazide, glimepiride), Meglitinides (Repaglinide and nateglinide), Biguanides (Metformin) Thiazolidinediones (rosiglitazone, pioglitazone), $\alpha$ Glucosidase inhibitors (acarbose, miglitol, voglibose) among these, metformin is the most common drug used. Metformin is given as monotheraphy with proper diet and exercise program and also with other medications to control high blood sugar. It is used in patients with type 2 diabetes. Controlling high blood sugar helps prevent kidney damage, blindness, nerve problems, loss of limbs, and sexual function problems. Proper control of diabetes reduces the risk of a heart attack or stroke. Metformin works by helping to restore the body's proper response to the insulin. It also decreases the amount of sugar that the liver makes and that the stomach/intestines absorb. Pioglitazone is a selective agonist drug, it belongs to Thiazolidinediones group .This drug activates PPAR $\gamma$ ( Peroxisome Proliferator activated receptor gamma ) receptors which indeed increases the transcription of insulinresponsive genes, thus controlling the glucose production, transportation and utilization. Hence this antidiabetic drug enhances the insulin sensitivity and reduces the glucose production via liver 
(hepatic gluconeogenesis) When the pre and the post blood sugar level were compared we found that, there was a significant reduction in fasting and post prandial blood sugar level with $\mathrm{p}$ value less than 0.05 . Hence pioglitazone reduces sugar level. Dyslipidemia is one of the risk factors for vascular complications in diabetic patients. According to study by Ploypun narindrarangkura, in 2019 cross sectional study to observe the prevalence of dyslipidemia associated with complications in 140,557 diabetic patients of average age 60 yrs. This study concluded that $88.9 \%$ have dyslipidemia. Dyslipidemia is a major risk factor for cardiovascular disease, stroke, and type 2 diabetes mellitus (T2DM) . But it is modifiable by lifestyle changes and medication. The disorder is characterized by an abnormal lipid profile, which can include elevated levels of plasma cholesterol, triglycerides, or both, or reduced levels of high-density lipoprotein cholesterol (HDL-C) .HDL level is indirectly proportional to the risk of coronary heart disease. It also shows atheroprotective actions. HDL cholesterol scavenges and eliminates LDL by transporting to liver and reprocessing takes place. HDL is also known as good cholesterol.LDL is also known as bad cholesterol. Presence of LDL is an indication of risk of coronary heart disease. LDL almost makes up the total body cholesterol. Increase in the LDL cholesterol build up on the walls of blood vessels resulting in plague. This plague narrows the blood vessel causing anginaThere was a significant decrease in the level of triglycerides with $\mathrm{p}<0.05$.There was also a significant increase in the HDL level with a pvalue $<0.05$. There is no significant change in the serum cholesterol and LDL Thus it is suggested that even though pioglitazone is an anti-diabetic drug, it is effective on the lipid profile. It reduces the triglycerides level All the study participants completed the study, there were no serious adverse drug reaction in the study participants. Limitations of our study were sample size was small and the study design should be a randomized comparative double blinded study but we have selected open labeled non comparative observational study and the study duration was also short. We suggest to conduct this study with large sample size, and with randomized double blinded design in multiple centres to know the full efficacy of the drug pioglitazone and its adverse effects so that it can be used in diabetic patients with dyslipidemia.

\section{CONCLUSION:}

The effect of pioglitazone on triglyceride level in type 2 diabetic patients was studied, it is evident that the Pioglitazone is both antidiabetic and antiatherogenic drug; we can conclude that the pioglitazone administration is effective to bring about significant change in the blood sugar, triglycerides.Also pioglitazone is a safe drug with less of side effects. , it will be more economical as well as increase patient compliance.

\section{REFERENCES}

1. Ahmed AM. History of diabetes mellitus. Saudi Med J 2002. Apr;23(4):373-378

2. Diabetes mellitus history- from ancient to modern times. Available at http://science. jrank.org/pages/2044/Diabetes-Mellitus htm

3. Patlak M. New weapons to combat an ancient disease: treating diabetes. FASEB J 2002. Dec;16(14): 185310.1096

4. Maitra A, Abbas AK. Endocrine system. In: Kumar V, Fausto N, Abbas AK (eds). Robbins and Cotran Pathologic basis of disease (7th ed) 2005. Philadelphia, Saunders; 1156-1226.

5. Chen L, Magliano DJ, Zimmet PZ. The worldwide epidemiology of type 2 diabetes mellitus: present and future perspectives. Nature reviews endocrinology. Available at: www.nature.com/uidfinder

6. Genetic basis of type 1 and type2 diabetes, obesity, and their complications. Advances and emerging opportunities in diabetes research: a Strategic Planning report of the DMICC.www.niddk.nih.gov/NR

7. SeemaAbhijeetKaveeshwar J. The current state of diabetes mellitus in India. PubMed Central (PMC). 2014

8. Joshi SR, Parikh RM. India - diabetes capital of the world: now heading towards hypertension. J Assoc Physicians India. 2007

9. Kumar A, Goel MK, Jain RB, Khanna P, Chaudhary V. India towards diabetes control: Key issues. Australas Med J. 2013.

10. DJ B. Effects of pioglitazone on lipid and lipoprotein metabolism. - PubMed -NCBI. 2007.

11. Ginsberg H.N., MacCallum P.R. The obesity, metabolic syndrome, and type 2 diabetes mellitus pandemic: Part I. Increased cardiovascular disease risk and the importance of atherogenic dyslipidemia in persons with the metabolic syndrome and type 2 diabetes mellitus. J CardiometabSyndr. 2009; 4(2):113-9.

12. Goldberg IJ Clinical review 124: Diabetic dyslipidemia: causes and consequences. J ClinEndocrinolMetab. 2001; 86(3):965-71. 